TRANSACTIONS OF THE

AMERICAN MATHEMATICAL SOCIETY

Volume 359, Number 12, December 2007, Pages 5991-6000

S 0002-9947(07)04160-8

Article electronically published on June 27, 2007

\title{
FILAMENT SETS, APOSYNDESIS, AND THE DECOMPOSITION THEOREM OF JONES
}

\author{
JANUSZ R. PRAJS AND KEITH WHITTINGTON
}

\begin{abstract}
Applications of the work introduced by the authors in a recent article, Filament sets and homogeneous continua, are given to aposyndesis and local connectedness. The aposyndetic decomposition theorem of Jones is generalized to spaces with the property of Kelley.
\end{abstract}

In 14, a line of research was initiated aimed at better understanding continua that lie strictly between being locally connected and indecomposable. Among other things, the resemblance of many of these spaces to indecomposable continua was made more transparent through the introduction of filament composants. The value of the notions introduced is also reflected in the number of classical results that are either strengthened or easily reproduced, and several such instances are given here.

Most of the results of this paper are for continua with a property introduced by Kelley in [8]. This is an important class of spaces. For instance, locally connected continua, hereditarily indecomposable continua, and homogeneous continua all have this property. A recent study [1] shows that absolute retracts for tree-like and for hereditarily unicoherent continua have this property. Spaces with this property have been very important in the study of hyperspaces, dating back to Kelley's original paper. This property is a mark indicating the regularity of the properties of a space. Henceforth, such spaces will be called Kelley spaces, or Kelley continua when appropriate.

The highlights of the paper include a generalization of Jones' aposyndetic decomposition theorem to Kelley continua, and several new results related to aposyndesis. Characterizations are given of aposyndesis and local connectedness in Kelley continua. In our decomposition theorem, it is not the $L(x)$ of Jones that partitions, but the maximal such. These continua are not necessarily terminal, but $x$ is an outlet point of $L(x)$. The decomposition is not necessarily continuous, but it is upper semi-continuous. The quotient space is aposyndetic.

\section{Preliminaries}

A continuum is a compact, connected, nonempty metric space. If $X$ is a continuum, $C(X)$ denotes the hyperspace consisting of all subcontinua of $X$ under the Hausdorff metric.

Received by the editors June 3, 2005 and, in revised form, August 31, 2005.

2000 Mathematics Subject Classification. Primary 54F15; Secondary 54H15.

Key words and phrases. Aposyndesis, aposyndetic, continuum, decomposition, Jones, Kelley, locally connected.

The first author was supported by the National Science Foundation grant DMS-0405374 and by the RCA assigned time award 2004/05 at California State University Sacramento.

(C)2007 American Mathematical Society

Reverts to public domain 28 years from publication 
Our definition of local connectedness follows the terminology of Kuratowski 11, p. 227]. A space is locally connected at a point $p$ if every neighborhood of $p$ contains a connected neighborhood of $p$. (Since we have made no requirement that the neighborhood be open, many authors would refer to this as being connected im kleinen at p.) A space is locally connected if it is locally connected at each of its points.

A metric space is called a Kelley space 1 provided that whenever points $x_{n}$ converge to an element $x$ contained in some subcontinuum $C$ of the space, there are continua $C_{n}$ containing $x_{n}$ that converge to $C$ under the Hausdorff metric. Every homogeneous continuum is a Kelley space [19].

Let $X$ be a topological space, $A$ and $B$ be subsets of $X$ such that $A$ is connected and $A \subset B$. The symbol $\operatorname{Cnt}_{B}(A)$ denotes the component of $B$ containing $A$. If $A$ is a singleton $\{a\}$, then $\operatorname{Cnt}_{B}(a)$ denotes $\operatorname{Cnt}_{B}(\{a\})$. Similarly, if $A$ is a continuum, $\operatorname{Ctu}_{B}(A)$ will denote the constituant of $B$ containing $A$. It equals the union of all continua in $B$ that meet $A$.

The following definitions were introduced in [14]. A continuum $C$ in $X$ is called filament (in $X$ ) provided there exists a neighborhood $N$ of $C$ (a set containing $C$ in its interior) such that $\operatorname{Cnt}_{N}(C)$ has empty interior. The continuum $C$ is called ample if for every open set $U$ containing $C$ there is a continuum $L$ such that $C \subset \operatorname{Int}(L) \subset L \subset U$. A set $S \subset X$ is called filament provided every continuum contained in $S$ is filament in $X$. The complement of a filament set will be called a co-filament set. By definition, a set is co-filament if and only if it intersects each ample continuum of the space. The following is known [14, Proposition 2.3].

Proposition 1.1. If $X$ is a Kelley continuum (i.e., a continuum which is also a Kelley space), then a subcontinuum $A$ of $X$ is ample if and only if $A$ is not filament.

If $p \in X$, the union of all filament continua in $X$ containing $p$ (if any) is called a filament composant of $X$ and is denoted $\mathrm{F} \operatorname{cs}(p)$. By [14, Proposition 1.8], $\operatorname{Fcs}(p)$ is first category in $X$.

\section{Aposyndesis AND LOCAL CONNECTEDNESS}

In this section we begin with some preliminary observations obtained by applying the concepts of filament and ample continua to questions of aposyndesis. This turns out to have special significance for Kelley continua and subsequently leads to characterizations of aposyndesis and local connectedness, and to some observations about cut points that relate interestingly to classical results.

A continuum $X$ is aposyndetic at $x$ with respect to $y$ if there exists a continuum $K \subset X \backslash\{y\}$ containing $x$ in its interior. It is aposyndetic at $x$ if it is aposyndetic at $x$ with respect to every other point, and aposyndetic if it is aposyndetic everywhere [5]. There are three set-functions: $L, K$, and $T$ that have been important in the theory of aposyndesis. We now define a fourth function $M$. Let $x \in X$ and $S \subset X$.

\footnotetext{
${ }^{1}$ Historically the name has varied. Originally called a space with Property 3.2 in [8], one also finds the expressions: space with the property of Kelley, space with property [k], etc.
} 
Then

$$
\begin{aligned}
K(x) & =\{y \in X \mid X \text { is not aposyndetic at } x \text { with respect to } y\} \\
L(x) & =\{y \in X \mid X \text { is not aposyndetic at } y \text { with respect to } x\} \\
T(S) & =\{y \in X \mid \text { every continuum with } y \text { in its interior meets } S\}, \\
M(x) & =\text { the intersection of all ample continua containing } x .
\end{aligned}
$$

Each of these sets is closed; $L(x)$ is a continuum [6]. When $S$ is connected, $T(S)$ is a continuum [3]. The members of the families $\{K(x) \mid x \in X\}$ and $\{L(x) \mid x \in X\}$ will be called, respectively, $K$-sets and $L$-sets.

The space $X$ is aposyndetic at $x$ if and only if $K(x)=\{x\}$. It is aposyndetic if $K(x)=\{x\}$ for each $x$, or equivalently, $L(x)=\{x\}$ for each $x$. Notice that $K(x)$ and $L(x)$ can be characterized as follows:

$$
\begin{aligned}
K(x) & =\{y \in X \mid \text { every continuum containing } x \text { in its interior contains } y\} \\
& =\bigcap\{D \in C(X) \mid x \in \operatorname{Int}(D)\} \\
L(x) & =\{y \in X \mid x \in K(y)\} \\
& =\{y \in X \mid \text { every continuum containing } y \text { in its interior contains } x\} .
\end{aligned}
$$

Notice further that:

$$
x \in K(y) \text { if and only if } y \in L(x) .
$$

Proposition 2.1. If $X$ is a continuum and $x \in X$, then $K(x) \subset M(x)$.

Proof. If $A$ is an ample continuum containing $x$, then $A$ is the intersection of the continua that contain $A$ in their interior, and this is a subcollection of those that contain $x$ in their interior. Thus $K(x) \subset A$. Since this holds for each ample continuum containing $x, K(x) \subset M(x)$.

Corollary 2.2. Let $X$ be a continuum and $x \in X$. If $\{x\}$ is the intersection of some collection of ample subcontinua of $X$, then $X$ is aposyndetic at $x$.

Proposition 2.3. Let $X$ be a continuum and $p, q \in X$. If there is some ample continuum $A$ in $X$ containing $p$ but not $q$, then $X$ is aposyndetic at $p$ with respect to $q$. If $X$ is a Kelley continuum, the converse is also true.

Proof. The forward implication is obvious. If $X$ is a Kelley continuum and $X$ is aposyndetic at $p$ with respect to $q$, then there is a continuum $D$ with $p$ in its interior such that $q \notin D$. The continuum $D$ is ample by Proposition 1.1 .

Proposition 2.4. If $X$ is a Kelley continuum and $x \in X$, then $K(x)=M(x)$.

Proof. By Proposition 2.1, $K(x) \subset M(x)$. For the reverse inclusion, let $y \in M(x)$. It suffices to show that $y$ is in every continuum containing $x$ in its interior.

If $C$ is a continuum containing $x$ in its interior, then $C$ is non-filament, and hence ample by Proposition 1.1. Since $y \in M(x), y \in C$.

Corollary 2.5. If $X$ is a Kelley continuum, then $X$ is aposyndetic at $x$ if and only if $\{x\}$ is the intersection of some collection of ample subcontinua of $X$. 
Corollary 2.6. Let $X$ be a Kelley continuum, $x, y \in X$, and $S$ a closed subset of $X$. Then

(1) $y \in K(x)$ if and only if every ample continuum that contains $x$ also contains $y$;

(2) $y \in L(x)$ if and only if every ample continuum that contains $y$ also contains $x$; and

(3) $y \in T(S)$ if and only if every ample continuum that contains $y$ intersects $S$.

Corollary 2.7. Let $X$ be a Kelley continuum and $x, y \in X$.

(1) If $y \in K(x)$, then $K(y) \subset K(x)$.

(2) If $y \in L(x)$, then $L(y) \subset L(x)$.

Proof. (1) If $y \in K(x)$, the collection of ample continua that contain $y$ contains the similar collection for $x$.

(2) Let $z \in L(y)$ and let $A$ be an ample continuum containing $z$. We use Corollary 2.6. Since $z \in L(y), y \in A$. Since $y \in L(x), x \in A$. Thus $z \in L(x)$.

The next result easily follows from (2.0.1) and the above observations.

Corollary 2.8. Let $X$ be a Kelley continuum and $x, y \in X$. The following statements are equivalent:

(a) $x \in K(y)$,

(b) $y \in L(x)$,

(c) $K(x) \subset K(y)$,

(d) $L(y) \subset L(x)$.

Proposition 2.9. Let $X$ be a Kelley continuum, and let $p$ and $q$ be distinct elements of $X$. Then $X$ is aposyndetic at $p$ with respect to $q$ if and only if $\mathrm{Ctu}_{X \backslash\{q\}}(p)$ is second category in $X$.

Proof. If $\mathrm{Ctu}_{X \backslash\{q\}}(p)$ is second category in $X$, then by [14, Proposition 1.8], it meets $X \backslash \operatorname{Fcs}(p)$. Thus there is a continuum $K$ in $X \backslash\{q\}$ containing $p$ as well as some point of $X \backslash \operatorname{Fcs}(p)$. Therefore, $K$ is non-filament and thus ample by Proposition 1.1. Hence $X$ is aposyndetic at $p$ with respect to $q$ by Proposition 2.3. The converse is obvious.

It should now be clear that much of the power in this new theory lies in the observation that when $X$ is a Kelley continuum, an ample continuum can often replace the role of a continuum with interior, and by [14, Proposition 1.8] and [14, Proposition 2.3], every continuum that contains a point $x$ and a point of the dense $G_{\delta}$ set $X \backslash \mathrm{F} \operatorname{cs}(x)$ is ample. The next two results demonstrate this by combining our theory with a condition for local connectedness given by H. S. Davis, 2, Theorem 4].

Proposition 2.10. Let $X$ be a Kelley continuum and $p \in X$. If

(1) for each $q \in X \backslash\{p\}, \operatorname{Ctu}_{X \backslash\{p\}}(q)$ is second-category in $X$, and

(2) for each subcontinuum $C$ with $p \notin C, \mathrm{Ctu}_{X \backslash C}(p)$ is second-category in $X$, then $X$ is locally connected at $p$.

Proof. By (1) and [14, Proposition 1.8], $\mathrm{Ctu}_{X \backslash\{p\}}(q)$ meets $X \backslash \mathrm{Fcs}(q)$. Hence, there is a continuum $D$ in $X \backslash\{p\}$ containing $q$ and a point of $X \backslash \operatorname{Fcs}(q)$. Hence, $D$ is 
non-filament, and thus ample by Proposition 1.1. By Corollary 2.6, it follows that $L(p)=\{p\}$. This is the first condition in [2, Theorem 4].

Similarly, by (2), for each continuum $C$ with $p \notin C$, there is a continuum $D$ such that $p \in \operatorname{Int}(D) \subset D \subset X \backslash C$. Thus $p \notin T(C)$, the remaining condition in Davis' theorem.

Corollary 2.11. Let $X$ be a Kelley continuum. Then $X$ is locally connected if and only if for each subcontinuum $C$ and each $p \in X \backslash C, \mathrm{Ctu}_{X \backslash C}(p)$ is second category in $X$.

The previous result relates to a bit of history. In [13, (17.8)] Maćkowiak and Tymchatyn answered a question from the University of Houston Mathematics Problem Book by showing that a continuum is locally connected if and only if it has Property A; that is (in the present terminology), if and only if for each subcontinuum $C, T(C)=C$. The latter was, in fact, earlier proved by Davis [2, Corollary 4.3]. Davis' proof was patterned after a proof given by Jones in 1941 [5, Theorem 8].

A point $q$ of a continuum $X$ such that $X \backslash\{q\}$ has more than one constituant is called a cut point of $X$. Please note that this terminology has a long and varied history. Some authors call this a weak cut point. Others use cut point for what we would call a separating point.

Corollary 2.12. If $X$ is a Kelley continuum, and $q \in X$ is not a cut point of $X$ (in other words, $X \backslash\{q\}$ is continuumwise connected), then for each $p \neq q, X$ is aposyndetic at $p$ with respect to $q$.

Corollary 2.13. Every Kelley continuum which is (somewhere) non-aposyndetic has at least one cut point.

The previous result contrasts interestingly with Jones' [ 6, Corollary], which states that a continuum which is not aposyndetic at any point must contain at least one cut point (and in fact, can have exactly one).

Jones also shows [6, Theorem 14] that if for each point $q$ of a continuum $X$, there is a point $p$ of $X$ such that $X$ is not aposyndetic at $p$ with respect to $q$, then the set of cut points of $X$ is dense in $X$. If we add to the hypothesis that $X$ is a Kelley space, then by Corollary 2.12 we obtain more.

Corollary 2.14. If $X$ is a Kelley continuum such that for each point $q$ of $X$ there is a point $p$ of $X$ such that $X$ is not aposyndetic at $p$ with respect to $q$, then every point of $X$ is a cut point.

A continuum is cyclically connected if each pair of points lies on a simple closed curve.

Corollary 2.15. Every cyclically connected Kelley continuum is aposyndetic.

\section{QUASI-ORDER STRUCTURES}

The results of the previous section lead in a natural way to quasi-order relations on Kelley continua. These relations, which will be used in the coming section to generalize Jones' aposyndetic decomposition, further reveal the regularity of properties of Kelley continua and highlight the duality between $L$-sets and $K$-sets.

Given a continuum $X$, we define a binary relation $\preceq$ in $X$ as follows. For any two points $x, y \in X$ we write $x \preceq y$ whenever $x \in K(y)$. Let $\preceq^{*}$ be the inverse relation 
to $\preceq$ on $X$. It follows by (2.0.1) that $x \preceq^{*} y$ if and only if $x \in L(y)$. Corollary 2.7 implies that if $X$ is a Kelley continuum, the relations $\preceq$ and ${ }^{*}$ are quasi-orders, i.e., they are reflexive and transitive.

Proposition 3.1. For every continuum $X$, the relations $\preceq$ and $\nwarrow^{*}$ are closed subsets of $X \times X$. Consequently, there exist minimal and maximal $K$-sets (L-sets).

Proof. Assume $\left(x_{n}, y_{n}\right)$ converges to $(x, y)$ in $X \times X$ with $x_{n} \preceq y_{n}$. Suppose there is a continuum $C \subset X$ such that $y \in \operatorname{Int}(C)$ and $x \notin C$. Consequently, $y_{n} \in \operatorname{Int}(C)$ and $x_{n} \notin C$ for almost all $n$, and thus $x_{n} \notin K\left(y_{n}\right)$ for almost all $n$, a contradiction. Hence such a $C$ does not exist, and $x \in K(y)$. The proof for $\preceq^{*}$ is similar.

The following result is a consequence of the quasi-order structure imposed on a Kelley continuum.

Corollary 3.2. Let $X$ be a Kelley continuum and $x \in X$. The following three conditions are equivalent:

(1a) $L(x)$ is a maximal L-set;

(2a) $K(x)$ is a minimal $K$-set;

(3a) $K(x)=\{y \in X \mid L(y)=L(x)\}$.

Similarly, the next three conditions are equivalent:

(1b) $L(x)$ is a minimal L-set;

(2b) $K(x)$ is a maximal $K$-set;

(3b) $L(x)=\{y \in X \mid K(y)=K(x)\}$.

\section{JONES' APOSYNDETIC DECOMPOSITION}

In 1955, F. B. Jones proved his celebrated aposyndetic decomposition theorem 7]. He showed that in a homogeneous continuum $X$, the sets $L(x)$ partition $X$ into mutually homeomorphic, homogeneous terminal continua; and furthermore, the decomposition is continuous, and the quotient space is aposyndetic. This theorem, and its subsequent improvements, have played a central role in the theory of homogeneous continua. In the early 1980s, J. T. Rogers, Jr. generalized and improved Jones' theorem in [15, [16] and [17. In the proof of [17, Theorem 5], Rogers still makes use of Jones' results that the $L(x)$ partition and are terminal continua. These facts will occur naturally as we show that Jones' decomposition nicely generalizes to Kelley continua.

Lemma 4.1. Let $X$ be a Kelley continuum. The following statements are equivalent.

(a) Aposyndesis is symmetric on $X$.

(b) For each $x \in X, L(x)=K(x)$.

(c) The L-sets partition $X$.

(d) The $K$-sets partition $X$.

Proof. By definition, the relation of being aposyndetic at one point with respect to another is symmetric if and only if for each $x, L(x)=K(x)$. Thus (a) and (b) are equivalent. If the $L$-sets partition $X$, then clearly the conditions $x \in L(y)$ and $y \in L(x)$ are equivalent. Thus (c) $\Rightarrow($ a). Similarly, (d) $\Rightarrow($ a). Thus it suffices to show that $(\mathrm{a}) \Rightarrow(\mathrm{c})$ and $(\mathrm{a}) \Rightarrow(\mathrm{d})$. 
Assume aposyndesis is symmetric. Then both (a) and (b) hold. If $x, y \in X$ and $y \in L(x)$, then by symmetry $x \in L(y)$. Thus $L(y) \subset L(x)$ and $L(x) \subset L(y)$ by Corollary 2.7. Thus (c) holds, and by (b), also (d).

If $C$ is a subcontinuum of a continuum $X$, a point $x \in C$ is called an outlet point of $C$ (comp. 9, [10]) if each continuum in $X$ meeting both $C$ and its complement contains $x$.

Proposition 4.2. If $X$ is a Kelley continuum, then for each $x \in X, x$ is an outlet point of the continuum $L(x)$.

Proof. Let $C$ be a continuum containing a point $y$ of $X \backslash L(x)$ and a point $q$ of $L(x)$. Suppose $x \notin C$. Since $y \notin L(x)$, there is an ample continuum $D$ in $X \backslash\{x\}$ containing $y$ by Corollary 2.6. The continuum $C \cup D$ is not filament and is thus ample by Proposition 1.1. Since this ample continuum does not contain $x$, we have $q \notin L(x)$ by Corollary 2.6, a contradiction.

Corollary 4.3. Let $X$ be a Kelley continuum and $x, y \in X$. If $L(x)$ intersects $L(y)$, then one is a subset of the other.

Proof. If we assume that $L(y)$ meets both $L(x)$ and its complement, then by Proposition 4.2 $x \in L(y)$. Thus $L(x) \subset L(y)$ by Corollary 2.7 .

A subcontinuum $C$ of a continuum $X$ is called a terminal continuum if the only continua in $X$ that meet $C$ are those that are either in $C$ or contain $C$.

Corollary 4.4. Let $X$ be a Kelley continuum. If the sets $L(x)$ partition $X$, they are terminal continua.

Proof. If the $L(x)$ partition $X$, then each $y \in L(x)$ is an outlet point of $L(x)$ since $L(x)=L(y)$. Thus each $L(x)$ is terminal.

Remark 4.5. We are now in a position to give a quick proof of Jones' theorem that in a homogeneous continuum, the sets $L(x)$ partition $X$ into terminal continua. A homogeneous continuum $X$ is a Kelley space, and thus, by Proposition 3.1, there is a minimal $L$-set, $L(x)$. By the homogeneity of $X$, every $L$-set is minimal. Thus by Corollary 4.3, the $L$-sets partition $X$. By Corollary 4.4 they are terminal.

By Jones' theorem (or the previous remark) together with Lemma 4.1]and Proposition 2.4 we immediately have the following.

Proposition 4.6. If $X$ is a homogeneous continuum, then for each $x \in X, L(x)=$ $K(x)=M(x)$.

Proposition 4.7. If $X$ is a continuum and $p \in X$ such that $L(p) \neq X$ and $p$ is an outlet point of $L(p)$, then $p$ is in the boundary of $L(p)$.

Proof. Suppose to the contrary that $p \in \operatorname{Int}(L(p))$. Let $C$ be any component of $\mathrm{Cl}(X \backslash L(p))$. Then $C$ meets $L(p)$ and its complement, yet does not contain $p$, contradicting that $p$ is an outlet point.

Proposition 4.8. Let $X$ be a Kelley continuum and $x, y \in X$. If $L(x)$ is a proper subset of $L(y)$, then $L(x)$ has empty interior with respect to $X$.

Proof. If $L(x)$ is a proper subset of $L(y)$, then $y \notin L(x)$ by Corollary 2.7. By definition, every subcontinuum of $L(y)$ with nonempty interior must contain $y$. 
We now study the properties of ample and filament continua with respect to monotone maps.

Proposition 4.9. Let $X$ be a Kelley continuum, $\mathcal{H}$ a monotone, upper semicontinuous decomposition of $X, \pi: X \rightarrow X / \mathcal{H}$ the natural map, $A$ a subcontinuum of $X$, and $\hat{A}$ a subcontinuum of $X / \mathcal{H}$. If $A$ is ample in $X$, then $\pi(A)$ is ample in $X / \mathcal{H}$. If $\hat{A}$ is ample in $X / \mathcal{H}$, then $\pi^{-1}(\hat{A})$ is ample in $X$.

Proof. First assume that $A$ is ample. Then $A \subset A_{1}=\pi^{-1}(\pi(A))$, so $A_{1}$ is also ample ( $A_{1}$ is a continuum since $\pi$ is monotone). Let $\hat{U}$ be a neighborhood of $\pi(A)$ in $X / \mathcal{H}$. Since $A_{1}$ is ample, there is a continuum $D$ with $A_{1} \subset \operatorname{Int}(D) \subset D \subset$ $\pi^{-1}(\hat{U})$. Since $\mathcal{H}$ is upper semi-continuous, there is a saturated open set $V$ such that $A_{1} \subset V \subset D$. It follows that $\pi(D)$ is a neighborhood of $\pi\left(A_{1}\right)=\pi(A)$. Since $\pi(D) \subset \hat{U}, \pi(A)$ is ample.

Now assume that $\hat{A}$ is ample in $X / \mathcal{H}$. Let $U$ be a neighborhood of $A_{1}=\pi^{-1}(\hat{A})$ in $X$. Since $A_{1}$ is saturated by elements of $\mathcal{H}$, and $\mathcal{H}$ is upper semi-continuous, there is a saturated open set $V$ such that $A_{1} \subset V \subset U$. Then $V=\pi^{-1}(\pi(V))$. Since $\pi(V)$ is a neighborhood of $\hat{A}$, there is a continuum $\hat{D}$ in $X / \mathcal{H}$ containing $\hat{A}$ in its interior and such that $\hat{D} \subset \pi(V)$. Since $\pi$ is monotone, the set $D=\pi^{-1}(\hat{D})$ is a continuum and satisfies $A_{1} \subset \operatorname{Int}(D) \subset D \subset V \subset U$. Hence, $A_{1}$ is ample.

Lemma 4.10. Let $X$ be a Kelley continuum, $\mathcal{H}$ a monotone, upper semi-continuous decomposition of $X, \pi: X \rightarrow X / \mathcal{H}$ the natural map, $\hat{F}$ a subcontinuum of $X / \mathcal{H}$, and $F=\pi^{-1}(\hat{F})$. Then $\hat{F}$ is filament in $X / \mathcal{H}$ if and only if $F$ is filament in $X$.

Proof. By Proposition 4.9, $F$ is ample if and only if $\hat{F}$ is ample. Since $X$ is a Kelley space, so is $X / \mathcal{H}$ by [19, Corollary 4.4]. Thus, in both spaces, a continuum is filament if and only if it is non-ample by Proposition 1.1. The result follows.

Proposition 4.11. Let $X$ be a Kelley continuum, and $\mathcal{H}$ a monotone, upper semicontinuous decomposition of $X$. Then $X / \mathcal{H}$ is locally connected at $C \in \mathcal{H}$ if and only if $C$ is ample in $X$, (in fact, if and only if it is locally connected at $C$ by open connected sets).

Proof. By [19, Corollary 4.4], $X / \mathcal{H}$ also a Kelley space. By [14, Corollary 2.4], $X / \mathcal{H}$ is locally connected at the point $C$ (in fact, locally connected by open connected sets) if and only if the singleton $\{C\}$ is ample in $X / \mathcal{H}$. Thus, the result follows by Proposition 4.9 .

Proposition 4.12. Let $X$ be a Kelley continuum and $\mathcal{H}$ a monotone, upper semicontinuous decomposition of $X$. The continuum $X / \mathcal{H}$ is indecomposable if and only if every $C \in \mathcal{H}$ is a co-filament set in $X$.

Proof. By [14, Proposition 1.9], $X / \mathcal{H}$ is indecomposable if and only if each singleton $\{C\}$ is co-filament in $X / \mathcal{H}$. Thus, it suffices to show that $\{C\}$ is co-filament in $X / H$ if and only if $C$ is co-filament in $X$. In turn, it suffices to show that $C$ meets every ample continuum in $X$ if and only if $C$ is an element of each ample subcontinuum of $X / \mathcal{H}$. This follows easily from Proposition 4.9 ,

Let $\mathcal{G}$ be the collection of maximal $L$-sets in a continuum $X$, i.e.,

$$
\mathcal{G}=\left\{L(x) \mid x \text { is maximal in } X \text { with respect to } \preceq^{*}\right\} \text {. }
$$


We conclude the paper with the following generalization of Jones' aposyndetic decomposition theorem for Kelley continua.

Theorem 4.13. If $X$ is a Kelley continuum, then $\mathcal{G}$ is an upper semi-continuous decomposition of $X$, and the quotient space $X / \mathcal{G}$ is aposyndetic and is also a Kelley continuum.

Moreover, $\mathcal{G}$ is the finest monotone, upper semi-continuous decomposition of $X$ such that the quotient space is aposyndetic; that is, for every monotone, upper semicontinuous decomposition $\mathcal{H}$ of $X$ with an aposyndetic quotient, the elements of $\mathcal{G}$ are subsets of elements of $\mathcal{H}$.

Proof. For $x, y \in X$, define $x \sim y$ provided there is an $L$-set $L(p)$ such that $x, y \in$ $L(p)$. By Corollary $4.3, \sim$ is an equivalence relation. Since $L$-sets are continua, the equivalence classes of $\sim$ are connected. Notice that $x \sim y$ if and only if for some $p \in x, x \preceq^{*} p$ and $y \preceq^{*} p$. Since $y \preceq^{*} p$ if and only if $p \preceq y$, it is seen that $\sim$ is the composition of $\preceq^{*}$ followed by $\preceq$. Since both $\preceq^{*}$ and $\preceq$ are closed subsets of $X \times X$ by Proposition 3.1, the relation $\sim$ is also closed. Consequently, the equivalence classes of $\sim$ are closed, and thus they are continua. Moreover, if $u_{n} \sim v_{n}$ and $\lim \left(u_{n}, v_{n}\right)=(u, v)$, then $u \sim v$, which implies the upper semi-continuity of the equivalence classes of $\sim$.

Let $C_{x}$ be the equivalence class of $x \in X$. Then $C_{x}$ is the closure of the union of the collection $\mathcal{L}_{x}$ of all $L$-sets containing $x$. By Corollary 4.3, this collection is linearly ordered by inclusion. Therefore, there are points $x_{n}$ such that $L\left(x_{n}\right) \in \mathcal{L}_{x}$, $L\left(x_{n}\right) \subset L\left(x_{n+1}\right)$, and $\operatorname{Lim} L\left(x_{n}\right)=C_{x}$. Let $p$ be an accumulation point of the sequence $\left\{x_{n}\right\}$, and let $q \in C_{x}$. There is a sequence $y_{n} \in L\left(x_{n}\right)$ such that $\lim y_{n}=q$. Therefore, $(p, q)$ is an accumulation point of $\left\{\left(x_{n}, y_{n}\right)\right\}$. Since $y_{n} \preceq^{*} x_{n}$ for each $n$, and $\preceq^{*}$ is closed, we have $q \preceq^{*} p$ and thus $q \in L(p)$. Hence, $C_{x}=L(p)$ and is a maximal $L$-set. Consequently, the equivalence classes of $\sim$ are precisely the members of $\mathcal{G}$.

To show that $X / \mathcal{G}$ is aposyndetic, let $\pi: X \rightarrow X / \mathcal{G}$ be the quotient map and $L\left(u_{1}\right), L\left(u_{2}\right)$ distinct elements of $\mathcal{G}$. Since $u_{1} \notin L\left(u_{2}\right)$, by Corollary 2.6 there is an ample continuum $A$ containing $u_{1}$ such that $u_{2} \notin A$. By Proposition 4.2, $A \cap L\left(u_{2}\right)=\emptyset$. Thus, by Proposition 4.9, $\pi\left(L\left(u_{1}\right) \cup A\right)$ is an ample continuum in $X / \mathcal{G}$ disjoint from $\pi\left(L\left(u_{2}\right)\right)$. Hence $X / \mathcal{G}$ is aposyndetic. The quotient space $X / \mathcal{G}$ is a Kelley continuum by [19, Corollary 4.4], since the decomposition is monotone.

To prove the last part of the theorem, suppose that $\mathcal{H}$ is a monotone, upper semi-continuous decomposition of $X$ such that some $G \in \mathcal{G}$ is not contained in any member of $\mathcal{H}$. Let $\pi_{\mathcal{H}}: X \rightarrow X / \mathcal{H}$ be the quotient map. Say $G=L(u)$, $u \in X$. Then there exists $v \in G$ such that $u_{1}=\pi_{\mathcal{H}}(u) \neq \pi_{\mathcal{H}}(v)=v_{1}$. Let $M_{1}$ be any continuum in $X / \mathcal{H}$ containing $v_{1}$ in its interior. Thus $M=\pi_{\mathcal{H}}^{-1}\left(M_{1}\right)$ is a continuum in $X$ containing $v$ in its interior. Since $X$ is not aposyndetic at $v$ with respect to $u$, we have $u \in M$. Consequently $u_{1} \in M_{1}$, and thus $X / \mathcal{H}$ is not aposyndetic at $v_{1}$ with respect to $u_{1}$. Hence $X / \mathcal{H}$ is not aposyndetic. The theorem is proved.

We make a couple of observations. First, note that each $K$-set is contained in an element of $\mathcal{G}$. Indeed, if $x, y \in K(z)$ for some $z$, then $z \in L(x)$ and $z \in L(y)$, which implies $x \sim y$. Secondly, it is easy to show that the elements of $\mathcal{G}$ are $T$-closed; that is, for each $L \in \mathcal{G}, T(L)=L$. 
Remark 4.14. Since $\mathcal{G}$ is the finest monotone, upper semi-continuous decomposition of $X$ such that $X / \mathcal{G}$ is aposyndetic, this is the core monotone aposyndetic decomposition guaranteed by FitzGerald and Swingle in [4, Theorem 2.7]. Thus this is also the decomposition given by McAuley in [12, and hence, the form taken by McAuley's decomposition for Kelley spaces is revealed. (See [18 for a synopsis.)

\section{REFERENCES}

1. J. J. Charatonik, W. J. Charatonik and J. R. Prajs, Arc property of Kelley and absolute retracts for hereditarily unicoherent continua, Colloq. Math. 97 (2003), 49-65. MR.2010542 (2004h:54028)

2. H. S. Davis, A note on connectedness im kleinen, Proc. Amer. Math. Soc. 19 (1968), 12371241. MR0254814 (40:8021)

3. H. S. Davis, D. P. Stadtlander and P. M. Swingle, Properties of the set functions $T^{n}$, Portugal. Mat. 21 (1962), 113-133. MR0142108(25:5501)

4. R. W. FitzGerald and P. M. Swingle, Core decomposition of continua, Fund. Math. 61 (1967), 33-50. MR 0224063(36:7110)

5. F. B. Jones, Aposyndetic continua and certain boundary problems, Amer. J. Math. 63 (1941), 545-553. MR0004771 (3:59e)

6. Concerning non-aposyndetic continua, ibid. 70 (1948), 403-413. MR0025161 (9:606h)

7. On a certain type of homogeneous plane continuum, Proc. Amer. Math. Soc. 6 (1955), 735-740. MR0071761 (17:180e)

8. J. L. Kelley, Hyperspaces of a continuum, Trans. Amer. Math. Soc. 52 (1942), 22-36. MR0006505 (3:315b)

9. P. Krupski, On homogeneous tree-like continua, Rend. Circ. Mat. Palermo (2), Suppl. 18 (1988), 327-336. MR0958744 (89g:54077)

10. P. Krupski and J. R. Prajs, Outlet points and homogeneous continua, Trans. Amer. Math. Soc. 318 (1990), 123-141. MR0937246 (90f:54054)

11. K. Kuratowski, Topology, Volume II, New edition, revised and augmented, Translated from French by A. Kirkor, Academic Press, New York-London, 1968. MR0259835 (41:4467)

12. L. F. McAuley, An atomic decomposition of continua into aposyndetic continua, Trans. Amer. Math. Soc. 88 (1958), 1-11. MR0124033 (23:A1353)

13. T. Maćkowiak and E. D. Tymchatyn, Continuous mappings on continua II, Dissertationes Math. (Rozprawy Mat.) 225 (1984). MR0739739 (87a:54048)

14. J. R. Prajs and K. Whittington, Filament sets and homogeneous continua, Topology Appl. 154 (2007), 1581-1591.

15. J. T. Rogers, Jr., Completely regular mappings and homogeneous, aposyndetic continua, Canad. J. Math. 33 (1981), 450-453. MR0617635 (83a:54012)

16. , Decompositions of homogeneous continua, Pacific J. Math. 99 (1982), 137-144. MR:0651491 (83c:54045)

17. Cell-like decompositions of homogeneous continua, Proc. Amer. Math. Soc. 87 (1983), 375-377. MR0681852 (84e:54040)

18. E. J. Vought, Monotone decompositions of continua, General topology and modern analysis, Academic Press, New York-London (1981) 105-113. MR0619036 (83b:54004)

19. R. W. Wardle, On a property of J. L. Kelley, Houston J. Math. 3 (1977), 291-299. MR0458379 $(56: 16582)$

Department of Mathematics and Statistics, California State University Sacramento, 6000 J Street, Sacramento, California 95819 - and - Institute of Mathematics, UniverSity of Opole, Ul. Oleska 48, 45-052 Opole, Poland

E-mail address: prajs@csus.edu

Department of Mathematics, University of the Pacific, Stockton, California 95211

E-mail address: kwhittin@pacific.edu 